\title{
RECONOCIMIENTO FACIAL DE EMOCIONES BÁSICAS Y SU RELACIÓN CON LA TEORÍA DE LA MENTE EN LA VARIANTE CONDUCTUAL DE LA DEMENCIA FRONTOTEMPORAL*
}

\author{
RECOGNITION OF BASIC EMOTION AND THEIR RELATION WITH THEORY OF MIND IN THE \\ BEHAVIORAL VARIANT OF FRONTOTEMPORAL DEMENTIA
}

\author{
María Eugenia Tabernero** y Daniel Gustavo Politis***
}

\begin{abstract}
*Trabajo realizado en el marco del subsidio Simulación motora y cognición social, su relación con los sistemas de neuronas en espejo. Estudio en pacientes con Demencia Frontotemporal (UBACYT 2011-2014) y del PIP Simulación mental en pacientes con Demencia Frontotemporal variante frontal (PIP 11220110100584-2012-2014) del Consejo Nacional de Investigaciones Científicas y Técnicas (CONICET), otorgados al Dr. Daniel Politis.

**Doctora en Psicología. Becaria Doctoral del Consejo Nacional de Investigaciones Científicas y Técnicas (CONICET) y Ayudante de primera en la Cátedra de Neuropsicología de la Facultad de Psicología de la Universidad de Buenos Aires (UBA). E-Mail: eugeniatabernero@yahoo.com.ar

***Doctor en Medicina y Médico Especialista Consultor en Neurología. Miembro de la Carrera del Investigador Científico del Consejo Nacional de Investigaciones Científicas y Técnicas (CONICET), Médico del Servicio de Neurología del HIGA EVA Perón y Profesor Asociado Regular de la Cátedra de Neuropsicología de la Facultad de Psicología de la Universidad de Buenos Aires (UBA).

E-Mail: dpolitis@psi.uba.ar
\end{abstract}

\section{RESUMEN}

Las emociones básicas están biológicamente determinadas y ligadas a conductas fundamentales para la supervivencia. Las emociones secundarias son aquellas que para su reconocimiento, requieren la elaboración cognitiva de un contexto social, por lo tanto, de la Teoría de la Mente (TdM). La TdM fue definida como la habilidad de conceptualizar estados mentales de otros individuos para explicar y predecir gran parte de su comportamiento. No es un concepto unitario, existen disociaciones entre los componentes cognitivo y el emocional de la TdM. Han sido establecidas las alteraciones en el reconocimiento facial de emociones básicas (RFEB) y en las tareas de TdM en la variante conductual de la demencia frontotemporal (DFTvc). El objetivo del trabajo que se informa fue estudiar el reconocimiento de emociones básicas o primarias y su relación con la TdM en pacientes con DFTve. El $81 \%$ de los pacientes mostró alteraciones en por lo menos uno de los tests de RFEB y el $35 \%$ en el reconocimiento de la prosodia emocional. El subtest Denominación y el Puntaje Total Emociones mostraron correlaciones con el Test Lectura de la Mente en los Ojos, mientras que todas las tareas de RFEB correlacionaron con la tarea de falsa creencia. Se encontraron dobles disociaciones entre TdM emocional y cognitiva, con mayor afectación del componente emocional. Como conclusión se corrobora la presencia de alteraciones en el RFEB con prosodia emocional conservada en la DFTvc. La ausencia de correlaciones entre emociones básicas y secundarias parecería indicar que se trata de procesos independientes entre sí. 
Palabras clave: Emociones básicas; Emociones secundarias; Teoría de la Mente emocional; Teoría de la Mente cognitiva; Demencia frontotemporal; Variante conductual.

\section{ABSTRACT}

Behavioral variant of frontotemporal dementia (bvFTD) is associated with dramatic changes in personality. The behavioral manifestations that show these patients may be due, to one hand, to abnormal emotional processing as a result of the anatomical regions concerned, and on the other, to alterations in Theory of Mind (ToM). Basic emotions are biologically determined. Joy, sadness, anger, disgust and fear are the emotional states that have received most agreement. ToM allows representing, inferring and interpreting mental and emotional states of others. It has been suggested that it is not a unitary concept. Cognitive Theory of Mind refers to the representations regarding the cognitive status of others. Affective ToM refers to the representation of emotional and motivational states. The objectives were to study the presence of alterations in the recognition of basic emotions in the face and voice in patients with bvFTD, as well as examine the relationships that exist between the recognition of basic emotions and TdM, to know if there are, or not, independent processes. To study, finally, the presence of dissociations between emotional and cognitive ToM, to know which one of these types of ToM shows greater alteration in our population. 26 bvFTD patients were assessed, diagnosed on the basis of the criteria proposed by the International Consortium Behavioral Variant FTD Criteria, and 23 control subjects. A battery for facial recognition of basic emotions (FRBE) was administered, selecting 60 photographs of the Pictures of Facial Affect. Three tasks were created, comprising 60 sheets each, 10 for every basic emotion. A test for recognition of emotional prosody was also administered. Among the ToM tests were Reading the Mind in the Eyes (RME), Faux Pas, and Firs Order False Belief Task (FOFBT). $81 \%$ of the patients showed alterations in at least one of the tests RFEB, and 35\% in emotional prosody recognition. All RFEB tasks showed a significantly moderate statistical correlation with the emotional prosody task. The Naming subtest and the Total Emotions Score (RFEB) showed correlations with RME test, while all tasks RFEB correlated with $\mathrm{FC} 1^{\circ} \mathrm{O}$. Finally, 10 simple dissociations between altered FRBE and preserved emotional prosody were found, and double dissociations between emotional and cognitive ToM, with greater impairment of the emotional component of ToM Discussion: A decreased performance is corroborated in the bvFTD patients group, relative to a control group, in all FRBE tasks. The recognition of emotional prosody is preserved. The presence of correlations between RME and all FRBE tasks may be explained by the neuropsychological structure of the tasks. The correlations found between RME and understanding of emotional prosody may be due to the fact that both tests assess emotional comprehension. As for the correlations found between $\mathrm{FC} 1^{\circ} \mathrm{O}$ and all FRBE tasks, taking into account that there seems to be no bibliographic outcome regarding to this correlation, that the neuropsychological tasks structure is completely different, and that $\mathrm{FC} 1^{\circ} \mathrm{O}$ showed no correlations with the emotional prosody task, it seems not possible for us to explain this result, leaving open the possibility for the development of a acceptable conclusion. Because of the absence of significant correlations between all of the recognition of basic and complex emotions tasks, we infer that the basic emotions are a qualitatively different kind of emotional state that secondary emotions, this lasts ones processed through ToM.

Key words: Basic emotions; Secondary emotions; Emotional Theory of Mind; Cognitive Theory of Mind; Behavioral variant of Frontotemporal dementia.

\section{INTRODUCCIÓN}

DEMENCIA FRONTOTEMPORAL VARIANTE CONDUCTUAL

La variante conductual de la Demencia Frontotemporal (DFTvc) se asocia a cambios drásticos en la personalidad del paciente y a alteraciones en la conducta social (Snowden, Austin, Sembi, Thompson, Craufurd \& Neary, 2008). El comienzo de los cambios en 
el comportamiento y la personalidad es insidioso, ya que incluye síntomas tales como agitación, depresión, desinhibición social y sexual, apatía, conductas de hiperfagia y de utilización (Mangone, Allegri, Arizaga \& Ollari, 2005; Muñoz, 2006; Neary, Snowden, Gustafson, Passant, Stuss \& Benson, 1998). Las alteraciones conductuales suelen aparecer antes que las cognitivas y se caracterizan por la presencia de un perfil frontal en la evaluación neuropsicológica, con alteraciones en tareas que evalúan la cognición social (CS) y las funciones ejecutivas (Adenzato, Cavallo \& Enrici, 2010; Adenzato \& Poletti, 2013; Snowden et al., 2008). Se pueden observar por otro lado, alteraciones en los aspectos semánticos del lenguaje. La memoria episódica verbal y visual, la visuoconstrucción y la visuoespacialidad se encuentran preservados en estadíos tempranos (Boxer \& Miller, 2005).

En la literatura se pueden hallar dos explicaciones acerca de las manifestaciones conductuales que caracterizan esta demencia. Ellas no son mutuamente excluyentes. Por una parte, se postula que las alteraciones conductuales que muestran estos pacientes sugieren un procesamiento emocional anormal como resultado de las regiones anatómicas afectadas en esta demencia (lóbulo frontal y en menor medida, amígdala) (Rosen, Pace-Savitsky, Perry, Kramer, Miller \& Levenson, 2004). Por la otra, los cambios observados en la personalidad resultarían de alteraciones en Teoría de la Mente (TdM) (Gregory et al., 2002; Lough, Kipps, Treise, Watson, Blair \& Hodges, 2006).

\section{EMOCIONES BÁSICAS Y SECUNDARIAS}

Las emociones básicas están biológicamente determinadas y ligadas a conductas fundamentales para la supervivencia; su comienzo es abrupto y su duración limitada. La expresión de cada emoción básica requiere activar ciertos músculos faciales que son los mismos en distintas culturas, de ahí su universalidad. Alegría, tristeza, enojo, asco y miedo son los estados emocionales que han recibido mayor acuerdo (Damasio, 1994, 2005; Ekman, 1999; LeDoux, 1999).

Las emociones secundarias son aquellas que para su reconocimiento, requieren la elaboración cognitiva de un contexto social. Surgen de variaciones sutiles y combinaciones de emociones básicas y son consideradas estados mentales (Baron-Cohen, Wheelwright \& Jolliffe, 1997; Ekman, 1999; Le Doux, 1999). Por lo tanto, es por medio de la cognición social (CS), y específicamente de la TdM, que se logra su reconocimiento.

\section{TEORÍA DE LA MENTE}

La Teoría de la Mente (TdM) permite representarse, inferir e interpretar los estados mentales (creencias, deseos, intenciones) y emocionales de los demás (Gallese \& Goldman, 1998). Se ha planteado que no es un concepto unitario sino que existen disociaciones entre los componentes cognitivo y emocional de la TdM. La TdM cognitiva refiere a las creencia acerca de las creencias, a las representaciones del estado cognitivo de los demás (Poletti, Enrici, Bonuccelli \& Adenzato, 2011; Shamay-Tsoory, Tomer, Berger, Goldsher \& Aharon-Peretz, 2005). La TdM afectiva hace referencia a la creencia acerca de los sentimientos, la representación de los estados afectivos y motivacionales (Shamay-Tsoory \& Aharon-Peretz, 2007; Shamay-Tsoory, Tomer, Berger, Goldsher \& Aharon-Peretz, 2005).

En cuanto a la evaluación neuropsicológica de los componentes afectivo y cognitivo de la TdM, existe acuerdo en describir las tareas de falsa creencia de primer y segundo orden como tareas de TdM cognitiva, ya que requieren la diferenciación entre la propia creencia y la del interlocutor (Shamay Tsoory, Harari, Aharon-Peretz \& Levkovitz, 2010), la detección de las metidas de pata (Faux Pas) como una tarea mixta que requiere representación tanto cognitiva como del estado emocional del otro (Baron-Cohen et al., 1999; Shamay-Tsoory et al., 2010), la detección de 
ironías como una tarea de alto componente emocional (Poletti, Enrici \& Adenzato, 2012; Shamay-Tsoory \& Aaron-Peretz, 2007) y la inferencia de estados emocionales a partir de la mirada como una tarea de TdM emocional (Poletti et al., 2011).

Se ha estudiado la presencia de disociaciones entre estos componentes en distintas poblaciones tales como pacientes con enfermedad de Parkinson (Bodden et al., 2010, Poletti et al., 2011), o esquizofrenia (Shamay-Tsoory \& Aharon-Peretz, 2007). En DFTve los resultados son contradictorios, no resultando posible el establecimiento de un patrón de afectación diferencial. Gregory y colaboradores (2002) encuentran afectados tanto el componente cognitivo como el emocional de la TdM. Por su parte, Torralva y colaboradores (2007) abordan las disociaciones entre TdM emocional y cognitiva en DFTve y si bien no administran tareas puras de TdM cognitiva, reportan alteraciones en una versión del test de lectura de la mente a partir de la mirada (Baron-Cohen, Jolliffe, Mortimore \& Robertson, 1997) -TdM emocional- y una diferencia significativa entre los componentes afectivo y cognitivo del Test Faux Pas (Stone, Baron-Cohen \& Kight, 1998) en favor de este último. Finalmente, Torralva, Roca, Gleichgerrcht, Bekinschtein y Manes (2009) administraron las mismas dos tareas que en el estudio anterior a otro grupo de pacientes con DFTve, resultando ambas alteradas, aunque no informan cuál de los componentes de la TdM muestra mayor afectación.

RECONOCIMIENTO DE EMOCIONES BÁSICAS EN LA VARIANTE CONDUCTUAL DE LA DEMENCIA FRONTOTEMPORAL

La herramienta más utilizada para su evaluación parece ser el set Pictures of Facial Affect (POFA - Ekman \& Friesen, 1976), compuesto por 110 fotografías de rostros de personas expresando seis estados emocionales básicos, alegría, tristeza, enojo, miedo, asco y sorpresa. Este set ofrece solo fotografías en blanco y negro, no cuenta con tareas ni normas, solo se muestra el porcentaje de acuerdo de un pequeño grupo de estudiantes universitarios norteamericanos en el reconocimiento de la emoción expresada para cada fotografía, lo que produce una gran variabilidad metodológica.

En DFTvc, Lough y colaboradores (2006) reportan alteraciones en el reconocimiento facial de emociones básicas (RFEB), empleando una tarea de denominación. Snowden y colaboradores (2008), empleando cuatro tareas de RFEB (tres de denominación y una de identidad emocional), encontraron alteraciones en esta población en relación con un grupo control para todas las tareas. Kumfor, Irish, Hodges y Piguet (2013) reportan alteraciones en el RFEB en DFTve, empleando dos tareas de denominación de estados emocionales básicos.

En cuanto al reconocimiento de emociones básicas, existen pocos estudios de voz en esta población, y la mayoría de ellos evalúa el reconocimiento de sonidos emocionales no verbales (por ejemplo, risas para la emoción alegría, arcadas para asco, etc.) y no la prosodia emocional. En Argentina y con normas locales se encuentra disponible el Subtest Prosodia Emocional - Comprensión del Protocolo para la Evaluación de la Comunicación de Montreal (MEC - Ferreres, Abusamra, Cuitiño, Côté, Ska \& Joanette, 2007). La prosodia emocional es definida como la trasmisión de los estados emocionales de un individuo a través de los aspectos suprasegmentales del habla. Keane, Calder, Hodges y Young (2002) estudiaron el reconocimiento de emociones en el rostro y en la voz (no prosodia emocional, sino sonidos que expresan emociones básicas) en DFTve y concluyen que la alteración es amodal, encontrando afectación en ambas modalidades de procesamiento emocional. Por su parte, Snowden y colaboradores (2008) sostienen que el reconocimiento de emociones básicas en el rostro y en la voz se encuentra alterado en esta demencia. Finalmente, Dara y colaboradores (2013) encuentran alteraciones para el reconocimiento de la prosodia emocional y el RFEB preservado en un estudio de caso único en DFTve. 
RELACIONES ENTRE EL RECONOCIMIENTO DE EMOCIONES BÁSICAS Y TDM

La presencia de correlaciones entre TdM y RFEB permitiría inferir si se trata de un mismo proceso cognitivo subyacente a ambos dominios. Sin embargo, hay pocos trabajos al respecto y con resultados contradictorios. Bora y colaboradores (2005) reportan correlación significativa entre una tarea de RFEB y una adaptación del Test Lectura de la Mente en los Ojos (Baron-Cohen, Wheelwright, Hill, Raste \& Plumb, 2001) en pacientes bipolares eutímicos. Aunque aclaran que la prueba empleada para la evaluación de emociones básicas no es sensible puesto que solo consta de 10 fotografías para los seis estados emocionales y sostienen que el reconocimiento de emociones básicas interviene en el proceso de reconocimiento emocional en el Test de Lectura de la Mente en los Ojos. Otro trabajo que reporta esta correlación es el de Henry y colaboradores (2009) que fue realizado con un grupo de pacientes con Esclerosis Múltiple, pero los autores no la explican. Brün (2005) evaluó el reconocimiento facial de emociones básicas y TdM y en pacientes esquizofrénicos, sin encontrar correlaciones entre las tareas. Por el contrario, Hall y colaboradores (2004) mencionan que encuentran correlaciones entre una tarea de atribución de estados emocionales complejos y RFEB en pacientes esquizofrénicos. Parece haber muy pocos trabajos en la literatura que aborden el RFEB, reconocimiento de prosodia emocional y TdM en DFTrc.

Dara y colaboradores (2013) en su estudio de caso único en una paciente con DFTvc, reportan una alteración selectiva para el reconocimiento de la prosodia emocional, con RFEB y tareas de empatía preservadas, aunque no realizan un análisis de la relación entre las tareas.

Por lo tanto, y a la luz de las investigaciones precedentes se espera encontrar alteraciones en todas las tareas de TdM y en todas las de reconocimiento emocional tanto en el rostro como en la voz en los pacientes con DFTvc. Las alteraciones en TdM son características de esta demencia y las alteraciones en el reconocimiento de emociones básicas parecen estar establecidas (Lough et al., 2006; Snowden et al., 2008; Kumfor et al., 2013). En cuanto a la relación entre el reconocimiento de emociones básicas y la TdM, se espera encontrar relaciones entre ambos dominios. En este punto, los antecedentes en la literatura son escasos, por lo tanto, su estudio resulta relevante.

\section{OBjetIVOS}

Los objetivos de la investigación realizada fueron: estudiar la presencia de alteraciones en el reconocimiento de emociones básicas en el rostro y en la voz en pacientes con DFTve, como así también estudiar si existen relaciones entre el reconocimiento de emociones básicas y la TdM, para conocer si se trata o no de procesos independientes entre sí. Por último, estudiar la presencia de disociaciones entre TdM emocional y cognitiva, para conocer cuál de estos tipos de TdM muestra mayor afectación en nuestra población.

\section{MÉTOdo \\ PARTICIPANTES}

Se evaluaron 26 pacientes del Laboratorio de Deterioro Cognitivo del HIGA Eva Perón con diagnóstico de DFTve en base a los criterios propuestos por el International Behavioural Variant FTD Criteria Consortium (Rascovsky et al., 2011).

La muestra estuvo integrada por 9 hombres y 17 mujeres, con una media de edad igual a $68 \pm 7$ años y de $6 \pm 3$ años de escolaridad. Todos presentaban alteraciones en por lo menos dos pruebas que evalúan CS, sin lesiones significativas en estudios de imágenes cerebrales estructurales (TAC, RMN) e hipoperfusión en el SPECT. Se consideraron como criterios de exclusión: antecedentes de enfermedades neurológicas, psiquiátricas y del desarrollo.

También se trabajó con un grupo control integrado por 23 sujetos (16 mujeres y 7 hombres), con una media de edad igual a $68 \pm 7$ 
años y una media de escolaridad de $6 \pm 2$ años que fueron seleccionados con los siguientes criterios de exclusión: antecedentes de enfermedades neurológicas, del desarrollo, trastornos psiquiátricos y un puntaje bruto inferior a 24 puntos en el Mini Mental State Examination (MMSE - Allegri et al., 1999; Folstein, M.F., Folstein, S.E. \& McHugh, 1975).

\section{INSTRUMENTOS}

Para el diagnóstico de demencia se utilizó una extensa batería neuropsicológica, que incluye los siguientes tests:

- Mini Mental State Examination (MMSEAllegri et al., 1999; Folstein et al., 1975). - Test del Reloj (Freedman, Learch, Kaplan, Winocur, Shulman \& Delis, 1994). - Tres subtests de la Batería Neuropsicológica Española (Artiola, Hermosillo, Heaton \& Pardee, 1999): California Verbal Learning Test, Fluencia Verbal Fonológica -P, M, R- y Dígitos Directos e Inversos.

- Test de Fluencia Verbal Semántica (animales) de Parkin (1999).

- Test de Denominación de Boston (Serrano et al., 2001).

- Wisconsin Card Sorting Test, 64 Card Version (Kangs, Thompson, Iverson \& Heaton, 2000).

- Trail Maiking Test A y B (Reitan \& Wolfson, 1985).

- Figura Compleja de Rey (Meyers, J. \& Meyers, K. 1995).

- Analogías, Matrices, Vocabulario y Diseño con Cubos de la Escala de Inteligencia para Adultos WAIS III (Wechsler, 2002).

PRUEBAS QUE EVALÚAN RECONOCIMIENTO FACIAL DE EMOCIONES BÁSICAS

Se seleccionaron 60 fotografías del Pictures of Facial Affect (POFA- Ekman \& Friesen, 1976) en base al trabajo de Calder y colaboradores (1996). Se armaron tres tareas, de 60 láminas cada una (180 láminas en total y 10 por emoción básica) -alegría, tristeza, enojo, asco, miedo y sorpresa-, utilizando el programa PowerPoint Office 2003 para Windows (Tabernero \& Politis, 2012) y las tareas son las siguientes:

Tarea de selección: se presentan seis fotografías por lámina, una por cada emoción básica. Se ubican en columna tres fotografías en la parte superior de la lámina y tres en la parte inferior. La consigna es "Elija la fotografía que muestra la emoción que se le indica oralmente". La tarea consiste en indicar verbalmente las emociones básicas, una por slide, que el examinado debe señalar entre los cinco distractores.

Tarea de apareamiento: se presentan siete fotografías por lámina, una por cada emoción básica, excepto para la emoción blanco que se repite en la fotografía de la izquierda. En columna se ubican hacia la derecha, tres fotografías en la parte superior de la lámina y tres en la parte inferior. Las fotografías de la izquierda están randomizadas de forma tal que lo que se repita con alguna de las fotografías de la derecha sea el estado emocional expresado y no el rostro de la persona. La consigna es "Indique cuál de las personas de la derecha siente lo mismo que la persona de la fotografía de la izquierda". El examinado debe aparear cada fotografía de la izquierda con aquella que exprese la misma emoción entre las fotografías de la derecha.

Tarea de denominación: se presenta una fotografía por lámina, acompañada de seis etiquetas que denominan los estados emocionales básicos. La fotografía se ubica en el centro de la lámina, y las palabras, en letra mayúscula de imprenta se disponen tres a la izquierda y tres a la derecha del blanco. El examinado debe seleccionar la etiqueta con el nombre de la emoción expresada en cada fotografía.

Puntuación: La respuestas se consignan manualmente. Se otorga un punto por respuesta correcta. El puntaje Total Emociones expresa la suma de aciertos en las tres tareas. Debido a la carencia de normas para esta batería, se utilizó un grupo control de sujetos sin 
patología, para darle confiabilidad y validez a los datos de los pacientes.

Dada la ausencia de criterios comunes en la selección de fotografías del set POFA (Ekman \& Friesen, 1976) para cada estado emocional, a excepción del trabajo de Calder y colaboradores (1996), se infornan en el Anexo 1 las fotografías utilizadas.

\section{Pruebas que eVALÚAN RECONOCIMIENTO DE EMOCIONES EN LA VOZ: PROSODIA EMOCIONAL - COMPRENSIÓN}

Para evaluar el reconocimiento de emociones en la voz se utilizó el Subtest de Prosodia Emocional - Comprensión que forma parte del Protocolo para la Evaluación de la Comunicación de Montreal (MEC) de Ferreres y colaboradores (2007). Consiste en cuatro pistas de audio que se reproducen en tres tonos emocionales básicos, alegría, tristeza y enojo. El paciente debe señalar cuál de los tres íconos dibujados expresa el tono emocional de cada frase.

\section{Pruebas que eVALÚAN LA TEORÍA DE LA MENTE}

- Falsa Creencia de Primero Orden $\left(\mathrm{FC} 1^{\circ} \mathrm{O}\right.$ Wimmer \& Perner, 1983) - TdM cognitiva.

- Faux Pas (Stone, Baron-Cohen \& Kight, 1998) - TdM cognitiva y emocional.

- Lectura de la Mente en los Ojos (LMO) (Baron-Cohen et al., 2001) - TdM emocional.

\section{Procedimiento}

El tipo de muestreo es no-probabilístico. La muestra quedó conformada por pacientes que se acercaron al Servicio de Neurología del HIGA Eva Perón y fueron derivados al Laboratorio de Deterioro Cognitivo. Allí se les administró la batería neuropsicológica para el diagnóstico de demencias detalladas, en dos sesiones de evaluación. Ante la sospecha de DFTve se administraron en otras dos sesiones las pruebas de CS, integradas por las pruebas que evalúan TdM ya detalla- das y el Iowa Gambling Task (IGT) - Bechara, Anderson, Damasio, A. \& Damasio, H. (1994). Luego del diagnóstico presuntivo de DFTve se administraron las pruebas específicas de emociones básicas.

Los sujetos del grupo control fueron seleccionados al azar entre conocidos de los miembros del equipo de trabajo y centros de jubilados. Como prueba de cribado se empleó el MMSE (Allegri et al., 1999; Folstein et al., 1975), considerando como patológico $y$, por ende, criterio de exclusión para el grupo control, un rendimiento inferior a 24 puntos.

\section{ANÁLISIS ESTADÍSTICO}

Se llevó a cabo un estudio de casos y controles. El análisis estadístico se realizó utilizando el programa Statistical Package for the Social Sciences (SPSS 18). Se empleó una prueba de diferencia de medias para comparar pacientes y controles en cuanto a edad y nivel educativo y con la prueba ji cuadrado se establecieron las diferencias por género. Para verificar la hipótesis de normalidad se empleó la prueba de Kolmogorov-Smirnov. Se obtuvieron medias y desvíos de rendimiento para cada tarea del grupo control, a partir de los cuales se calcularon los puntajes $Z$ para el grupo de pacientes en todas las tareas de RFEB.

Se empleó la prueba ANOVA para comparar las medias de rendimiento entre los grupos en las tareas de RFEB, y $r$ de Pearson para medir la correlación entre variables. Empleando cálculos porcentuales se obtuvieron niveles de sensibilidad y especificidad para las tareas de RFEB. Se buscaron además, disociaciones y dobles disociaciones entre tareas.

\section{Resultados}

La Tabla 1 presenta los estadísticos descriptivos (edad, escolaridad, género) de ambos grupos en las tareas de RFEB. No se observan diferencias significativas entre ambos grupos para las tres variables. 
La distribución de los puntajes resultó normal para el grupo de pacientes y para el grupo control (ver Tabla 2).

El rendimiento de los pacientes en los tests neuropsicológicos que componen la batería diagnóstica se muestra en la Tabla 3 , en la que se observa mayor afectación del Test de Vocabulario de Boston (Serrano et al., 2001) y el Trail Making Test A y B (Reitan \& Wolfson, 1985), y el $73 \%$ de los pacientes empleando estrategia IV (patológica) para la copia de la Figura Compleja de Rey (Meyers, J. \& Meyers, K., 1995).

Un total de 21 sobre 26 pacientes evaluados mostró alteraciones en por lo menos dos tareas de RFEB. Esto constituye el 81\% de la muestra. Empleando la prueba ANOVA se comparó el rendimiento de pacientes y controles en las tareas de RFEB. Se observan diferencias significativas para cada tarea, a favor del grupo control (ver Tabla 4).

La Tabla 5 muestra las medias y los desvíos estándar del rendimiento en ambos grupos para todas las tareas de RFEB. A partir del rendimiento de los sujetos del grupo control en cada tarea, se calculó el rendimiento de los pacientes en puntaje $Z$.

El 35\% de los pacientes, es decir, 9 de ellos, mostró alteraciones en el reconocimiento de la prosodia emocional. La media de rendimiento fue igual a $8.54( \pm 2.64)$ para la muestra de pacientes. Por último, el $80.76 \%$ de los pacientes mostró un rendimiento patológico en el Test LMO, el $61.53 \%$ en el Test Faux Pas, y el $42.3 \%$ en $\mathrm{FC}^{\circ} \mathrm{O}$.

Empleando $r$ de Pearson se correlacionó el rendimiento de los pacientes en tareas de reconocimiento de emociones básicas. Todas las tareas de RFEB mostraron una correlación estadística significativamente moderada con la Tarea Comprensión de la Prosodia Emocional (Tarea Selección $r=.461, p=.018$; Tarea Apareamiento $r=.426, p=.030$; Tarea Denominación $r=.417, p=.034$; Total Emociones $r=.467, p=.016)$. Utilizando el mismo estadístico se correlacionó también el rendimiento en estas tareas con las de TdM. LMO mostró una correlación significativamente moderada con la Tarea Denominación $(r=.597, p=.001)$, con el puntaje Total
Emociones $(r=.460, p=.018)$ y con la prueba de Comprensión de la Prosodia Emocional $(r=.428, p=.029)$. Falsa Creencia de Primer Orden mostró correlaciones moderadas con todas las tareas de RFEB (Tarea Selección $r=.462, p=.020$; Tarea Apareamiento $r=.482, p=.015$, Tarea Denominación $r=.551, p=.004$; Total Emociones $r=.536, p=.006$ ), pero no con Comprensión de la Prosodia Emocional $(r=.371$; $p=.068)$. Faux Pas no mostró correlaciones con las tareas de reconocimiento de emociones básicas (Selección $r=.165, p=.431$; Apareamiento $r=.160, p=.444$, Denominación $r=.339, p=.097$; Total Emociones $r=.230, p=.269$, Comprensión de la prosodia emocional $r=-.050, p=.811$ ).

Empleando cálculos porcentuales se obtuvieron niveles de sensibilidad y especificidad para las tareas de RFEB. La batería muestra una sensibilidad del 76.92\% y una especificidad del 95.7\%, teniendo en cuenta las tres pruebas de RFEB más el puntaje Total Emociones.

Se encontraron 10 disociaciones simples entre tareas de RFEB alteradas y Comprensión de la Prosodia Emocional conservada (ver Tabla 6). No se observa la disociación complementaria. Entre tareas de reconocimiento emocional y TdM, no es posible hallar dobles disociaciones debido a que se consideró criterio diagnóstico la alteración en por lo menos dos pruebas de CS. No obstante, sin embargo existen disociaciones simples en 5 pacientes que presentan alteraciones en TdM y reconocimiento de emociones básicas en el rostro y en la voz preservadas.

Finalmente se observan dobles disociaciones entre tareas de TdM emocional y cognitiva: 10 pacientes con $\mathrm{LMO}$ alterada y $\mathrm{FC} 1^{\circ} \mathrm{O}$ conservada, y dos pacientes con la disociación complementaria. Faux Pas, que es considerada una prueba mixta, mostró disociaciones con ambos componentes de la TdM.

\section{Discusión}

El principal objetivo de este trabajo fue estudiar la presencia de alteraciones en el reco- 
nocimiento de emociones básicas en el rostro y en la voz en pacientes con DFTve, intentando conocer si existen relaciones entre las alteraciones en el reconocimiento emocional y la TdM.

Se corrobora un rendimiento disminuido en el grupo de DFT-ven, en relación a un grupo control, en todas las tareas de RFEB. Estos hallazgos están en consonancia con la literatura (Kumfor, Irish, Hodges \& Piguet, 2013; Lough et al., 2006; Snowden et al., $2008)$. Un alto porcentaje de pacientes $(81 \%)$ mostró alteraciones en por lo menos una de las tres pruebas que evalúan reconocimiento de emociones básicas en el rostro. Si bien se trata de una muestra pequeña, el número de 26 pacientes supera en cantidad a grupos de pacientes estudiados y reportados en la literatura. Se ha estudiado el RFEB empleando distintas tareas, ya que si bien Denominación es la más utilizada en la bibliografía citada, esta tarea puede resultar sencilla debido a la limitada cantidad de etiquetas que describen cada emoción básica. La batería mostró buenos niveles de sensibilidad y especificidad.

En cuanto al reconocimiento de la prosodia emocional, solo el 35\% de los pacientes presentó alteraciones en este dominio. El bajo porcentaje de pacientes que muestra alterado el rendimiento en la prueba Prosodia Emocional - Compresión puede deberse a que se trata de una tarea corta que evalúa el reconocimiento de tres de los seis estados emocionales básicos, en comparación con las tareas que evalúan reconocimiento de emociones en rostros que son más extensas y completas. Si bien son escasos los estudios que investigan a la vez el reconocimiento de emociones básicas en el rostro y en la voz, la presencia de alteraciones en ambas modalidades ha sido reportada en distintas poblaciones. Nuestros hallazgos no corroboran los reportados por Dara y colaboradores (2013) en su estudio de caso único en una paciente con DFTvc en el que se encontró una alteración selectiva para el reconocimiento de la prosodia emocional, y que en nuestra muestra se encuentra conservada. Por otro lado, Keane y colabora- dores (2002) y Snowden y colaboradores (2008) sostienen que la alteración en el reconocimiento emocional en la DFTve es amodal; sin embargo en la muestra estudiada, el bajo porcentaje de pacientes que presentan dificultades para el reconocimiento de emociones en la voz no permite afirmar que se encuentre alterada esta modalidad de reconocimiento.

La totalidad de las tareas de RFEB mostró una correlación significativa con el test de prosodia emocional. No se hallaron en la literatura trabajos que estudien esta correlación. Una explicación posible para esta correlación es el hecho de que todas las tareas involucradas evalúan comprensión emocional. La ausencia de dobles disociaciones entre las tareas de RFEB y la comprensión de la prosodia emocional indicarían que se trata de procesos relacionados pero que pueden alterarse selectivamente.

A su vez, nos propusimos estudiar las relaciones entre el reconocimiento de emociones básicas y la TdM emocional y cognitiva. Se hallaron solo algunas correlaciones entre tareas de RFEB reconocimiento de emociones básicas y de TdM emocional. En primer lugar, el test LMO (Baron-Cohen et al., 2001) mostró correlaciones con la prueba Denominación de emociones básicas. Dado que ambas tareas evalúan denominación de estados emocionales y teniendo en cuenta que LMO (Baron-Cohen et al., 2001) no mostró correlaciones con las otras dos tareas de RFEB, la correlación hallada se explicaría por la estructura neuropsicológica de las pruebas. En segundo lugar, la prueba LMO (Baron-Cohen et al., 2001) mostró correlación significativa con el Subtest Prosodia Emocional - Comprensión. Considerando que el $80.76 \%$ de los pacientes que integran la muestra DFTve mostró alteraciones en LMO (Baron-Cohen, et al., 2001) mientras que el reconocimiento la prosodia emocional se encuentra conservado, la explicación más plausible para esta correlación sería que la misma se debe a que ambas pruebas evalúan comprensión emocional. Finalmente, Faux Pas (Stone et al., 1998) no mostró correlación con ninguna de 
las pruebas de reconocimiento de emociones básicas. Por lo tanto, al no haberse hallado correlaciones significativas en la totalidad de las tareas involucradas para reconocimiento de emociones básicas y complejas, no es posible concluir que las emociones básicas están relacionadas con las secundarias. Inferimos entonces que las emociones básicas son un tipo cualitativamente distinto de estado emocional que las emociones secundarias, procesadas a través de la TdM.

Todas las tareas de RFEB mostraron correlación con la tarea de TdM cognitiva Falsa creencia de primer orden (Wimmer \& Perner, 1983). Este test es considerado un instrumento de evaluación de la TdM cognitiva en tanto carece de componente emocional. Por lo tanto arribar a una conclusión o intentar explicar esta fuerte correlación entre tareas no resulta posible. Teniendo en cuenta que parece no haber antecedentes bibliográficos para este resultado, que la estructura de las tareas es completamente distinta, y que $\mathrm{FC} 1^{\circ} \mathrm{O}$ (Wimmer \& Perner, 1983) no mostró correlaciones con la tarea de comprensión de la prosodia emocional, no resulta posible explicar este resultado, quedando abierta la elaboración de una conclusión que resulte aceptable.

Por último, nos propusimos estudiar la presencia de disociaciones entre TdM emocional y cognitiva, con el objetivo de conocer cuál de estos dos tipos muestra mayor afectación en nuestra población. Se encontraron dobles disociaciones entre las tareas que evalúan ambos componentes de TdM, encontrándose más afectado en esta población el componente emocional, con una tasa más alta de rendimiento patológico para el test LMO (Baron-Cohen et al., 2001) (80.76\%). La prueba Faux Pas (Stone et al., 1998) mostró disociaciones con ambas tareas. Hasta el momento no tenemos conocimiento acerca de la existencia de otro trabajo que aborde puntualmente la presencia de estas disociaciones en una muestra rioplatense de pacientes con DFTrc, o que incluso estudie cuál de los componentes de la TdM muestra mayor afectación en esta enfermedad. Torrava y colaboradores (2007) encuentran diferencias significativas entre una tarea de TdM emocional y el componente cognitivo del Test Faux Pas (Stone et al., 1998) en DFTvc, a partir de lo cual concluyen que existirían disociaciones dentro de la TdM. En cuanto a las disociaciones entre emociones básicas y TdM, se observan disociaciones simples, 5 pacientes con alteraciones en $\mathrm{TdM}$ y reconocimiento de emociones básicas en el rostro y en la voz preservadas. No encontramos antecedentes en la literatura que analicen esta disociación. Las interpretaciones al respecto se ven sesgadas por el hecho de que se considera como criterio diagnóstico la presencia de alteraciones en por lo menos dos pruebas de CS, lo cual redunda en alguna tarea de $\mathrm{TdM}$ siempre afectada.

En síntesis, la mayoría de los pacientes presentó alteraciones en el RFEB, por lo tanto los pacientes con DFTve muestran alteraciones en el reconocimiento de emociones básicas en el rostro. La ausencia de correlaciones entre emociones básicas y secundarias, más la presencia de disociaciones simples, parecerían indicar que se trata de procesos que son independientes entre sí.

Dadas las limitaciones de la población estudiada, estos hallazgos no son generalizables, por lo tanto resultaría de interés ampliar esta muestra, llevar a cabo estudios similares que corroboren los resultados encontrados y estudiar estas correlaciones en otras patologías (enfermedad de Parkinson, Asperger, trastorno bipolar, etc.). 
Emociones básicas y teoría de la mente en la demencia frontotemporal

TABLA 1

ESTADÍSTICOS DESCRIPTIVOS DE AMBOS GRUPOS

\begin{tabular}{|l|c|c|cc|c|}
\hline & $\begin{array}{l}\text { DFTvc } \\
n=26 \\
M(D E)\end{array}$ & $\begin{array}{c}\text { Grupo control } \\
n=23 \\
M(D E)\end{array}$ & $\begin{array}{c}\text { Comparación de } \\
\text { Medias }\end{array}$ & \\
\hline Edad & $68(7)$ & $68(7)$ & .663 & .511 & \\
Escolaridad en años & $6(3)$ & $6(2)$ & -.388 .700 & \\
\hline Género Femenino & 17 & 16 & & & .042 \\
& 9 & 7 & & & \\
\hline
\end{tabular}

TABLA 2

PRUeba de NORMalidAd de LOS PUNTAJES CORRESPONDIENTES A LAS TAREAS DE RFEB

\begin{tabular}{|l|cc|cc|}
\hline \multicolumn{1}{|c|}{ Tareas } & \multicolumn{2}{|c|}{ DFTvc } & \multicolumn{2}{c|}{ Grupo control } \\
& $Z$ & $p$ & $Z$ & $p$ \\
\hline Selección & .668 & .763 & .643 & .802 \\
Apareamiento & .391 & .998 & .615 & .843 \\
Denominación & .815 & .521 & .640 & .798 \\
Total Emociones & .559 & .914 & .807 & .547 \\
\hline
\end{tabular}


TABLA 3

Rendimiento del GRUPO dE PACIENTES EN LA batería PARA DIAGNÓStico dE DFTVC

\begin{tabular}{|c|c|c|c|}
\hline \multicolumn{2}{|c|}{ Test } & \multirow{2}{*}{$\begin{array}{c}M \\
25.81\end{array}$} & \multirow{2}{*}{$\begin{array}{c}D E \\
\pm 3.39\end{array}$} \\
\hline Mini-Mental State Examination & & & \\
\hline Test del Reloj & & 11.81 & \pm 2.28 \\
\hline California Verbal Learning Test & $\begin{array}{l}\text { Lista A } 1^{\circ} \text { Ensayo } \\
\text { Total Aprendizaje } \\
\text { Lista B } \\
\text { Recuerdo Inmediato } \\
\text { Recuerdo Inmediato con Pistas } \\
\text { Recuerdo Diferido } \\
\text { Recuerdo Diferido con Pistas } \\
\text { Reconocimiento }\end{array}$ & $\begin{array}{l}-.62 \\
-1.28 \\
-.54 \\
-1.25 \\
-.98 \\
-.77 \\
-.57 \\
-.51\end{array}$ & $\begin{array}{r} \pm .66 \\
\pm 1.04 \\
\pm .66 \\
\pm 1.08 \\
\pm 1.18 \\
\pm 1.08 \\
\pm 1.06 \\
\pm 1,19\end{array}$ \\
\hline Fluencia verbal fonológica & & -1.08 & \pm .63 \\
\hline Dígitos directo & & -.10 & \pm .88 \\
\hline Dígitos inverso & & -.75 & \pm .98 \\
\hline Fluencia semántica & & -1.03 & \pm .89 \\
\hline Boston & & -1.95 & \pm 1.40 \\
\hline Wisconsin Card Sorting Test & $\begin{array}{l}\text { Respuestas Perseverativas } \\
\text { Respuestas Nivel Conceptual }\end{array}$ & $\begin{array}{l}-1.29 \\
-1.38\end{array}$ & $\begin{array}{l} \pm 1.19 \\
\pm .76\end{array}$ \\
\hline TMT & $\begin{array}{l}\text { Parte A } \\
\text { Parte B } \\
\text { Copia }\end{array}$ & $\begin{array}{l}-3.14 \\
-3.62 \\
-2.62\end{array}$ & $\begin{array}{l} \pm 2.12 \\
\pm 2.45 \\
\pm 3.04\end{array}$ \\
\hline Figura Compleja de Rey & $\begin{array}{l}\text { Tiempo } \\
\text { Recuerdo Inmediato } \\
\text { Recuerdo Diferido } \\
\text { Reconocimiento } \\
\text { Estrategia de Copia }\end{array}$ & $\begin{array}{c}-.57 \\
-1.39 \\
-1.62 \\
-.93 \\
\text { IV el } 73 \%\end{array}$ & $\begin{array}{c} \pm 1.21 \\
\pm .91 \\
\pm .97 \\
\pm 1.32 \\
\text { acientes }\end{array}$ \\
\hline WAIS III-R & $\begin{array}{l}\text { Analogías } \\
\text { Matrices } \\
\text { Vocabulario } \\
\text { Cubos }\end{array}$ & $\begin{array}{l}-1.41 \\
-.87 \\
-.79 \\
-1.13\end{array}$ & $\begin{array}{l} \pm .65 \\
\pm .70 \\
\pm .46 \\
\pm .63\end{array}$ \\
\hline
\end{tabular}

Nota: Para las tareas Mini Mental State Examination y el Test del Reloj, el rendimiento está expresado en puntaje bruto. Para el resto de las tareas, en puntaje Z. Para la estrategia de copia de la Figura Compleja de Rey se empleó porcentaje por tratarse de una variable escalar. 


\section{TABLA 4}

ANOVA ENTRE PACIENTES Y CONTROLES PARA LAS TAREAS DE RECONOCIMIENTO FACIAL DE EMOCIONES BÁSICAS

\begin{tabular}{|c|c|c|c|c|c|c|}
\hline \multicolumn{2}{|c|}{ Tareas } & $S C$ & $d f$ & $M C$ & $F$ & $p$ \\
\hline Selección & $\begin{array}{l}\text { Entre Grupos } \\
\text { Intragrupos } \\
\text { Total }\end{array}$ & $\begin{array}{c}662.701 \\
1663.977 \\
2326.678\end{array}$ & $\begin{array}{c}1 \\
57 \\
58\end{array}$ & $\begin{array}{c}662.701 \\
29.193\end{array}$ & 22.701 & .000 \\
\hline Apareamiento & $\begin{array}{l}\text { Entre Grupos } \\
\text { Intragrupos } \\
\text { Total }\end{array}$ & $\begin{array}{l}1031.756 \\
2970.346 \\
4002.102\end{array}$ & $\begin{array}{c}1 \\
57 \\
58\end{array}$ & $\begin{array}{c}1031.756 \\
52.111\end{array}$ & 19.799 & .000 \\
\hline Denominación & $\begin{array}{l}\text { Entre Grupos } \\
\text { Intragrupos } \\
\text { Total }\end{array}$ & $\begin{array}{c}731.926 \\
1900.752 \\
2632.678\end{array}$ & $\begin{array}{c}1 \\
57 \\
58\end{array}$ & $\begin{array}{c}731.926 \\
33.347\end{array}$ & 21.949 & .000 \\
\hline Total Emociones & $\begin{array}{l}\text { Entre Grupos } \\
\text { Intragrupos } \\
\text { Total }\end{array}$ & $\begin{array}{c}7211.079 \\
15393.599 \\
22604.678\end{array}$ & $\begin{array}{c}1 \\
57 \\
58\end{array}$ & $\begin{array}{c}7211.079 \\
270.063\end{array}$ & 26.701 & .000 \\
\hline
\end{tabular}

TABLA 5

MEDIAS Y DESVíOS DE RENDIMIENTO EN LAS TAREAS DE RECONOCIMIENTO FACIAL DE EMOCIONES BÁSICAS EN AMBOS GRUPOS

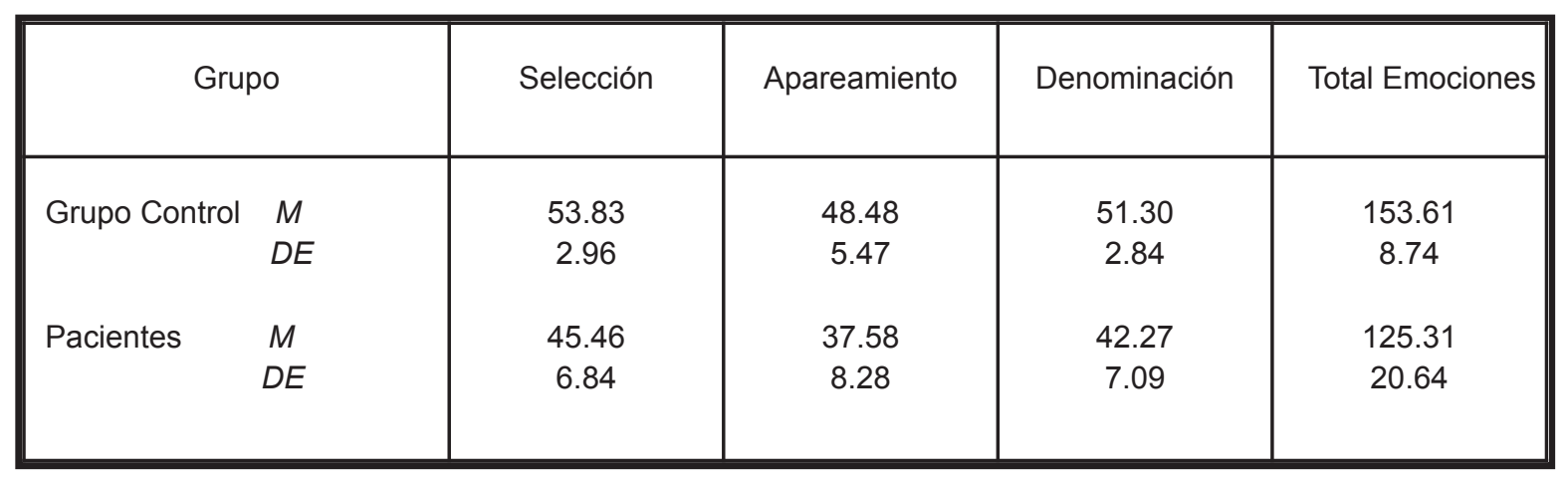




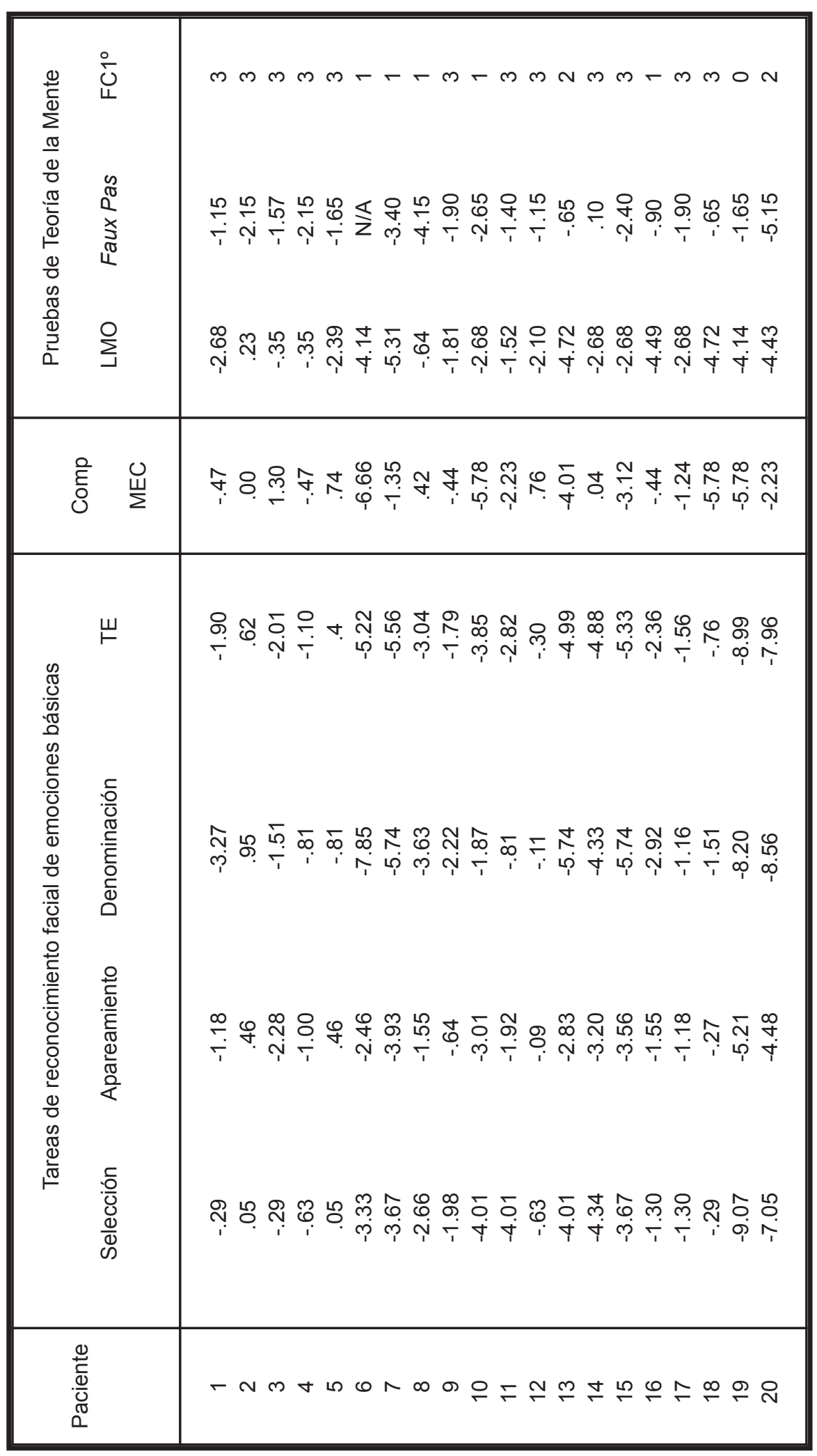


Emociones básicas y teoría de la mente en la demencia frontotemporal

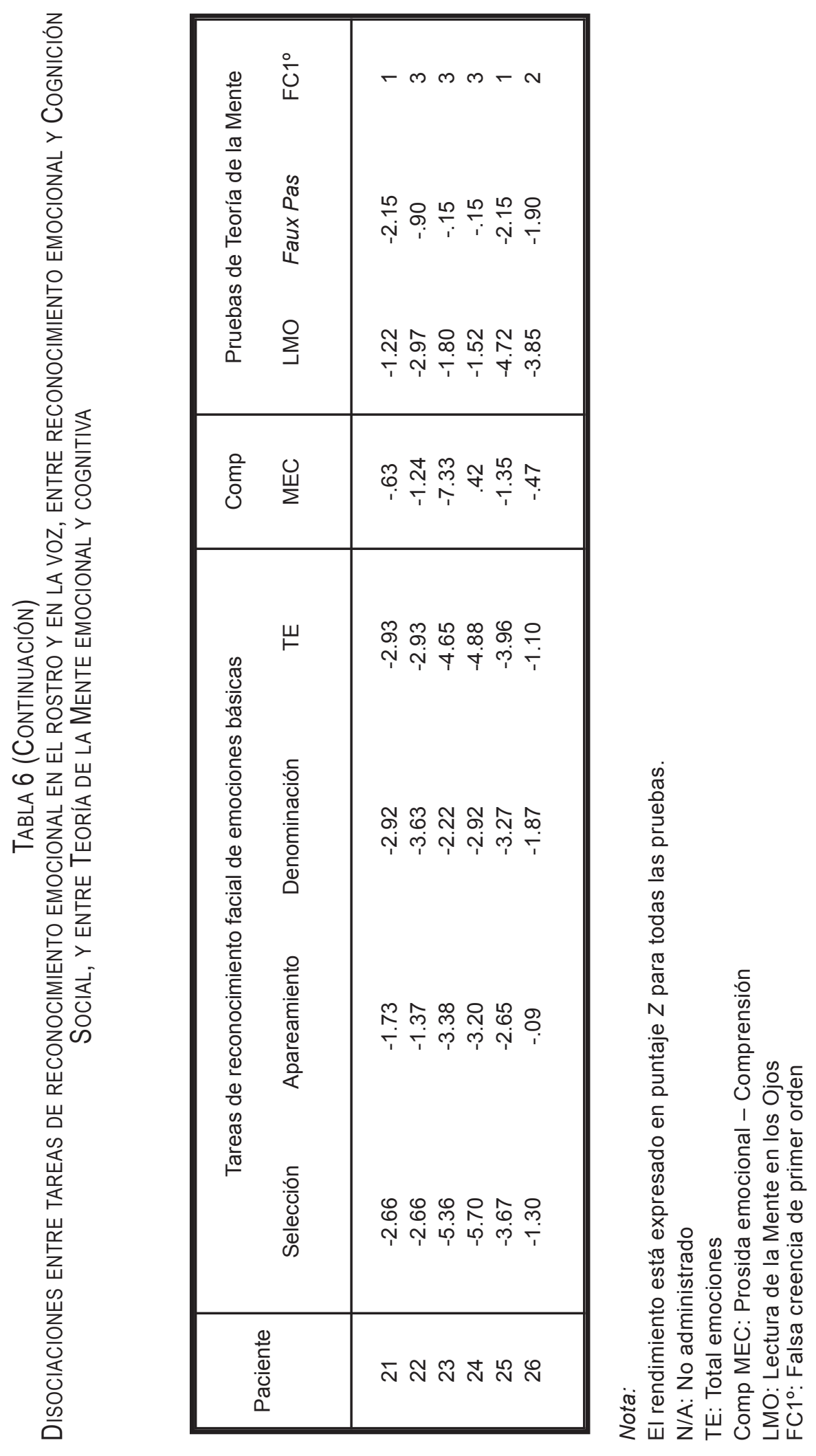




\section{ANEXO 1 \\ Fotografías del POFA (EKMAN \& FRIESEN, 1976) EMPLEAdAS EN LAS TAREAS DE RECONOCIMIENTO FACIAL DE EMOCIONES BÁSICAS}

Alegría: C-2-18, EM-4-07, JJ-4-07, MF-1-06, MO-1-04, NR-1-06, PE-2-12, PF-1-06, SW-3-09, WF-2-12

Asco: C-1-04, EM-4-17, JJ-3-20, MF-2-13, MO-2-18, NR-3-29, PE-4-05, PF-1-24, SW-1-30, WF-3-11

Enojo: C-2-12, EM-5-14, JJ-3-12, MF-2-07, MO-2-11, NR-2-07, PE-2-21, PF-2-04, SW-4-09, WF-3-01

Miedo: C-1-23, EM-5-21, JJ-5-13, MF-1-26, MO-1-23, NR-1-19, PE-3-21, PF-2-30,SW-2-30, WF-3-16

Sorpresa: C-1-10, EM-2-11, JJ-4-13, MF-1-09, MO-1-14, NR-1-14, PE-6-02, PF-1-16, SW-1-16, WF-2-16

Tristeza: C-1-18, EM-4-24, JJ-5-05, MF-1-30, MO-1-30, NR-2-15, PE-2-31, PF-2-12, SW-2-16, WF-3-28

\section{REFERENCIAS BIBLIOGRÁFICAS}

Adenzato, M. \& Poletti, M. (2013). Theory of mind abilities in neurodegenerative diseases: An update and a call to introduce mentalizing tasks in standard neuropsychological assessments. Clinical Neuropsychiatry, 10(5), 223-234.

Adenzato, M., Cavallo, M. \& Enrici, I. (2010). Theory of mind ability in the behavioural variant of frontotemporal dementia: An analysis of the neural, cognitive, and social levels. Neuropsychologia, 48, 2-12. http://dx.doi.org/ 10.1016/j.neuropsychologia.2009.08.001

Allegri, R.F., Ollari, J.A., Mangone, C.A., Arizaga, R.L., De Pascale, A., Pellegrini, M. et al. (1999). El "Mini-Mental State Examination" en la Argentina: Instrucciones para su administración [The "Mini-Mental State Examination" in Argentina: Instructions for administration]. Revista Neurológica Argentina, 24(1), 1-5.

Artiola, L., Hermosillo, D., Heaton, R.K. \& Pardee, R.E. (1999). Manual de normas y procedimientos para la Batería Neuropsicológica en español [Manual of rules and procedures for the neuropsychological battery in Spanish]. Tucson, Arizona: $m$ Press.

Baron-Cohen, S., Jolliffe, T., Mortimore, C. \& Robertson, M. (1997). Another advanced test of theory of mind: Evidence from very high functioning adult with autism or Asperger Syndrome. Journal of Child Psychology and Psychiatry, 38, 813-822.

Baron-Cohen, S., Ring, H.A., Wheelright, S., Bullmore, E.T., Brammer, M.J., Simmons, A. et al. (1999). Social intelligence in the normal and autistic brain: An fMRI study. European Journal of Neuroscience, 11,1891-1898.http:// dx.doi.org/10.1046/j.1460-9568.1999.00 621.x

Baron-Cohen, S., Wheelwright, S., Hill, J., Raste, Y. \& Plumb, I. (2001). The 'Reading the Mind in the Eyes' Test Revised Version: A study with normal adults, and adults with Asperger Syndrome or High-Functioning Autism. Journal of Child Psychology and Psychiatry, 42, 241-252.

Baron-Cohen, S., Wheelwright, S \& Jolliffe, T. (1997). Is there a "Language of the Eyes"? Evidence from normal adults, and adults with 
autism or asperger syndrome. Visual Cognition, 4(3), 311-331. http://dx.doi.org/10.1080/71375 6761

Bechara, A., Anderson, S.W., Damasio, A. \& Damasio, H. (1994). Insensitivity to future consequences following damage to the human prefrontal cortex. Cognition, 50, 7-15.

Bodden, M., Mollenhauer, B., Trenkwalder, C., Cabanel, N., Eggert, K. M., Unger, M. et al. (2010). Affective and cognitive theory of mind in patients with Parkinson's disease. Parkinsonism and Related Disorders, 16, 466-470.

Bora, E., Vahip, S., Gonul, A.S., Akdeniz, F., Alkan, M., Ogut, M. \& Eryavuz, A. (2005). Evidence for theory of mind deficits in euthymic patients with bipolar disorder. Acta Psychiatrica Scandinavica, 112, 110-116. http://dx.doi.org/10.111 1/j.1600-0447.2005.0 0570.x

Boxer, A. \& Miller, B. (2005). Clinical features of frontotemporal dementia. Alzheimer Disease and Associated Disorders, 19(1), 3-6.

Brün, M. (2005). Emotion recognition, 'theory of mind' and social behavior in schizophrenia. Psychiatry Research, 133, 135-147. http://dx. doi.org/10.1016/j.psychres.2004.10.007

Calder, A.J., Young, A.W., Rowland, D., Perrett, D.I., Hodges, J.R. \& Etcoff, N.L. (1996). Facial emotion recognition after bilateral amygdala damage: Differentially severe impairment of fear. Cognitive Neuropsychology, 13(5), 699745. http://dx.doi.org/10.1080/026432996381 890

Damasio, A. (1994). El error de Descartes. La razón de las emociones [Descartes' error: Emotion reason]. Santiago de Chile, Chile: Andrés Bello.

Damasio, A. (2005). En busca de Spinoza. Neurobiología de la emoción y los sentimientos [Looking for Spinoza: Joy, sorrow, and the feeling]. Barcelona, España: Crítica.

Dara, C., Kirsch-Darrow, L., Ochfeld, E., Slenz, J., Agranovich, A., Vasconcellos-Faria, A. et al. (2013). Impaired emotion processing from vocal and facial cues in frontotemporal dementia compared to right hemisphere stroke. Neurocase, 19(6), 521-529. http://dx.doi.org/10.108 $0 / 13554794.2012 .701641$
Ekman, P. (1999). Basic emotions. En T. Dalgleish \& M. Power, (Eds.), Handbook of cognition and emotion (pp. 45-60). Sussex, U.K.: Wiley. http://dx.doi.org/10.1002/ 0470013494

Ekman, P. \& Friesen, W.V. (1976). Pictures of facial affect. Palo Alto, CA: Consulting Psychologists Press.

Ferreres, A., Abusamra, V., Cuitiño, M., Côté, H., Ska, B. \& Joanette, Y. (2007). Protocolo MEC. Protocolo para la evaluación de la Comunicación de Montreal. Versión en español [MEC protocol. Protocol for Evaluation of Communication of Montreal. Spanish version]. Buenos Aires: Neuropsi.

Folstein, M.F., Folstein, S.E. \& McHugh, P.R. (1975). 'Mini-mental state'. A practical method for grading the cognitive state of patients for the clinician. Journal of Psychiatry Research, 12, 189-198.

Freedman, M., Learch, K., Kaplan, E., Winocur, G., Shulman, K. \& Delis, D. (1994). Clock drawing: A neuropsichological analysis. New York: Oxford University Press.

Gallese, V. \& Goldman, A. (1998). Mirror neurons and the simulation theory of mindreading. Trends in Cognitive Sciences, 2(12), 493-501.

Goodglass, H. \& Kaplan, E. (1986). Test de Vocabulario de Boston: La evaluación de la afasia $y$ trastornos relacionados [Boston Vocabulary Test: The assessment of afasia and related disorder] (2da. ed). Madrid: Editorial Médica Panamericana.

Gregory, C., Lough, S., Stone, V., Erzinclioglu, S., Martin, L., Baron-Cohen, S. et al. (2002). Theory of mind in patients with frontal variant frontotemporal dementia and Alzheimer's disease: Theoretical and practical implications. Brain, 125, 752-764. http://dx.doi.org/10.10 93/brain/awf079

Hall, J., Harris, J., Sprengelmeyer, R., Sprengelmeyer, A., Young, A., Santos, I. et al. (2004). Social cognition and face processing in schizophrenia. British Journal of Psychiatry, 185, 169-170. http://dx.doi.org/10.1192/bjp.185.2. 169 
Henry, J., Phillips, L., Beatty, W., McDonald, S., Longley, W., Joscelyne, A. et al. (2009). Evidence for deficits in facial affect recognition and theory of mind in multiple sclerosis. Journal of the International Neuropsychological Society, 15, 277-285. http://dx.doi.org/ 10.1017/S1355617709090195

Kangs, S., Thompson, L., Iverson, G. \& Heaton, R. (2000). Wisconsin Card Sorting Test 64 Card Version: Professional Manual. Odessa, FL: Psychological Assessment Resources.

Keane, J., Calder, A., Hodges, J. \& Young, A. (2002). Face and emotion processing in frontal variant frontotemporal dementia. Neuropsychologia, 40, 655-665. http://dx.doi.org/10.10 16/ S0028- 3932(01)00156-7

Kumfor, F., Irish, M., Hodges, J. \& Piguet, O. (2013). Discrete neural correlates for the recognition of negative emotions: Insights from frontotemporal dementia. PLoS ONE, 8(6), e67 457. http://dx.doi.org/10.1371/journal. pone. 00 67457

LeDoux, J. (1999). El cerebro emocional [The emotional brain]. Argentina: Editorial Planeta.

Lough, S., Kipps, C., Treise, C., Watson, P., Blair, J. \& Hodges, J. (2006). Social reasoning, emotion and empathy in frontotemporal dementia. Neuropsychologia, 44, 950-958.

Mangone, C., Allegri, R., Arizaga, R. \& Ollari, J. (2005). Demencia. Enfoque multidisciplinario [Dementia. Multidisciplinary focus] (1ra ed.). Argentina: Polemos.

Meyers, J. \& Meyers, K. (1995). Rey Complex Figure Test and Recognition Trial. Florida, USA: Psychological Assessment Resources.

Muñoz, D. (2006). Sintomatología de las demencias frontotemporales. En R. Alberca \& S. López-Pousa (Eds.), Enfermedad de Alzheimer $y$ otras demencias [Alzheimer's Desease and other dementias] (pp. 33-42) (3ra. ed.). Madrid: Editorial Médica Panamericana.

Neary, D., Snowden, J.S., Gustafson, L., Passant, U., Stuss, D. \& Benson, D.F. (1998). Frontotemporal lobar degeneration: A consensus on clinical diagnostic criteria. Neurology, 51, 15461554..http://dx.doi.org/10.1212/WNL.51.6.154
Parkin A. (1999). Exploraciones en neuropsicología cognitiva [Explorations in Cognitive Neuropsychology]. Madrid: Editorial Médica Panamericana.

Poletti, M., Enrici, I. \& Adenzato, M. (2012). Cognitive and affective theory of mind in neurodegenerative diseases: Neuropsychological, neuroanatomical and neurochemical levels. Neuroscience and Biobehavioral Reviews, 36, 2147-2164. http://dx.doi.org/10.10 16/j.neubiorev.2012.07.004

Poletti, M., Enrici, I., Bonuccelli, U. \& Adenzato, M. (2011). Theory of mind in Parkinson's disease. Behavioural Brain Research, 219, 342350. http://dx.doi.org/10.1016/j.bbr.2011.01.0 10

Rascovsky, K., Hodges, J., Knopman, D., Méndez, M., Kramer, J., Neuhaus, J. \& Miller, B. (2011). Sensitivity of revised diagnostic criteria for the behavioural variant of frontotemporal dementia. Brain, 134(9), 2456-2477. http://dx. doi. org/10.1093/brain/awr179

Reitan, R. \& Wolfson, D. (1985). The HalsteadReitan Neuropsychological Test Battery. Tucson, Arizona: Neuropsychology Press.

Rosen, H., Pace-Savitsky, K., Perry, R., Kramer, J., Miller, B. \& Levenson, R. (2004). Recognition of emotion in the Frontal and Temporal Variants of Frontotemporal Dementia. Dementia and Geriatric Cognitive Disorders, 17, 277-281. http://dx.doi.org/10.1159/000077154

Serrano, C.M., Allegri, R.F., Drake, M., Butman, J., Harris, P., Nagle, C. \& Ranalli, C. (2001). Versión corta en español del Test de Denominación de Boston: Su utilidad en el diagnóstico diferencial de la enfermedad de Alzheimer [Reducid Spanish version of Boston naming test: Its usefulness in the differential diagnose of Alzheimer's disease]. Revista Neurológica Argentina, 33(7), 624-627.

Shamay-Tsoory, S.G. \& Aharon-Peretz, J. (2007). Dissociable prefrontal networks for cognitive and affective theory of mind: A lesion study. Neuropsychologia, 45, 3054-3067. http://dx. doi.org/10.1016/j.neuropsychologia.2007.05. 021 
Shamay-Tsoory, S.G., Harari, H., Aharon-Peretz, J. \& Levkovitz, Y. (2010). The role of the orbitofrontal cortex in affective theory of mind deficits in criminal offenders with psychopathic tendencies. Cortex, 46, 668-677. http:// dx.doi.org/10.1016/j.cortex.2009.04.008

Shamay-Tsoory, S.G., Shur, S., Barcai-Goodman, L., Medlovich, S., Harari, G. \& Levkovitz, Y. (2007). Dissociation of cognitive from affective components of theory of mind in schizophrenia. Psychiatry Research, 149, 11-23. http://dx. doi.org/10.1016/j.psychres. 2005. 10.018

Shamay-Tsoory, S.G., Tomer, R., Berger, B.D., Goldsher, D. \& Aharon-Peretz, J. (2005). Impaired "affective theory of mind" is associated with right ventromedial prefrontal damage. Cognitive and Behavioral Neurology, 18(1), 55-67.

Snowden, J., Austin, N., Sembi, S., Thompson, J., Craufurd, D. \& Neary, D. (2008). Emotion recognition in Huntington's disease and frontotemporal dementia. Neuropsychologia, 46, 2638-2649. http://dx.doi.org/10.1016/j.neuro psychologia.2008.04.018

Stone, V., Baron-Cohen, S. \& Kight R. (1998). Frontal lobe contributions to theory of mind. Journal of Cognitive Neuroscience, 10(5), 640656. http://dx.doi.org/10.1162/089892998562 942
Tabernero, M.E. \& Politis, D. (2012). Evaluación del reconocimiento facial de emociones básicas en Demencia Frontotemporal variante frontal [Evaluation of facial recognition of basic emotions in frontal variant frontotemporal dementia]. Revista Argentina de Neuropsicología, 20, 24-34.

Torralva, T., Kipps, C., Hodges, J., Clark, L., Bekinschtein, T., Roca, M., Calcagno, M. \& Manes, F. (2007). The relationship between affective decision-making and theory of mind in the frontal variant of fronto-temporal dementia. Neuropsychologia, 45, 342-349.

Torralva,T., Roca, M., Gleichgerrcht, E., Bekinschtein, T. \& Manes, F. (2009). Neuropsychological battery to detect specific executive and social cognitive impairments in early frontotemporal dementia. Brain, 132, 1299-1309.

Wechsler, D. (2002). Wechsler Adult Intelligences Scale - third edition. San Antonio, TX: The Psychological Corporation.

Wimmer, H. \& Perner, J. (1983). Beliefs about beliefs: Representation and the containing function of wrong beliefs in young children's understanding of deception. Cognition, 13, 103128. http://dx.doi.org/10.1016/0010-0277(83) 90 004-5

\author{
Laboratorio de Deterioro Cognitivo \\ Hospital Interzonal General de Agudos Eva Perón \\ Consejo Nacional de Investigaciones Científicas \\ y Técnicas (CONICET) \\ Buenos Aires - República Argentina
}

Fecha de recepción: 13 de noviembre de 2014 Fecha de aceptación: 18 de enero de 2016 